\title{
Efficient adaptive phase field simulation of directional solidification of a binary alloy
}

\author{
C.W. Lan ${ }^{\mathrm{a}, \mathrm{b}, *}$, Y.C. Chang ${ }^{\mathrm{a}}$ \\ a Department of Chemical Engineering, National Taiwan University, Taipei 10617, Taiwan, ROC \\ ${ }^{\mathrm{b}}$ Institute of Advanced Material Study, Kyushu University, 6-1 Kasuga Koen, Kasuga 816-8580, Japan \\ Received 6 September 2002; accepted 29 November 2002 \\ Communicated by Dr. D.T.J. Hurle
}

\begin{abstract}
Efficient adaptive phase field simulation based on a finite volume method is carried out to study the morphological development during directional solidification of a nickel/copper alloy. The adaptive nature of the method allows the calculation to cover different length scales for the interface, solute diffusion, and heat conduction. With the frozen temperature approximation, our calculated results are in reasonable agreement with previous ones (J. Crystal Growth 200 (1999) 583). However, the use of a much larger domain allows us to perform simulation at low speed near the onset of constitutional supercooling, where both solutal boundary layer and cell wavelength are large. For the same domain size, the calculated results without using the frozen temperature approximation remain about the same, even though the release of latent heat lowers the steady interface position and the thermal gradient in the melt side.
\end{abstract}

(C) 2003 Elsevier Science B.V. All rights reserved.

PACS: $68.70 .+\mathrm{w} ; 81.30 . \mathrm{Fb}$

Keywords: A1. Computer simulation; A1. Dendrites; A1. Directional solidification; A1. Heat transfer; A1. Segregation; A1. Solidification

\section{Introduction}

In directional solidification of alloys, the onset of morphological instability and the following pattern development are fundamental and important problems, which have attracted extensive research for the past 40 years. The classic

\footnotetext{
*Corresponding author. Department of Chemical Engineering, National Taiwan University, Taipei 10617, Taiwan, ROC. Fax: + 886-223633917.

E-mail address: cwlan@ntu.edu.tw (C.W. Lan).
}

Mullins-Sekerka instability [1] provides the first approach to predict the onset cellular wavelength from a steady-state planar interface. Warren and Langer [2] further accounted the initial transient to the Mullins-Sekerka analysis, and with some success, to predict the primary spacing from the initial instability to a steady array. Although the theory for morphological instability has made some significant progress, the detailed pattern formation, being a highly nonlinear problem, and its prediction still rely on numerical simulation. However, the geometrical complexities have 
discouraged the use of front tracking approaches $[3,4]$. The recently developed alloy phase-field simulation [5-9] seems to be useful in this category of the problem, and some promising examples have been demonstrated by Boettinger and Warren [9] recently.

By using the frozen temperature approximation (FTA), Boettinger and Warren [9] made the first attempt to simulate the pattern formation at high speed during directional solidification of a nickel/ copper system by modifying their binary phase field model [5-8]. Reasonable agreement with the classic theory was found near the absolute stability limit (at high speed). Some important phenomena, such as solute trapping and tip splitting, were also illustrated. Because the thermal boundary layer was not considered due to FTA and the thinner solutal boundary layer, at high speed, as well as the smaller cell wavelength, they were able to use a small domain for simulation by using a structured grid. However, under the restrictions inherent in using a structured mesh, numerical studies of phenomena at lower speeds and truly non-isothermal effects including the heat of fusion, both having large solutal and thermal boundary layers, become very difficult. In this report, we present an efficient adaptive phase field simulation for the same problem. Due to the adaptive nature of the method, the domain size, which is only limited by computer memory, can be extremely large covering both the length scales for thermal and solutal boundary layers, while the minimum cell size remains small enough for the diffusive interface. Although, as will be discussed shortly, it is still not enough to resolve all the problems, the present simulation may shed a light to a more realistic simulation in the future. In the following section, the phase field model and the adaptive finite volume method are described briefly. Section 3 is devoted to results and discussion, followed by conclusions and comments in Section 4.

\section{Phase field simulation and adaptive finite volume method}

For comparison purposes, the model employed in Refs. $[9,10]$ is adopted here. Similar to the treatment by Boettinger and Warren [9], the equations are recast into a moving frame with constant speed $V$. From the observer point of view, the system can been regarded as a domain having continuous feeding from the top and flowing out from the bottom, as illustrated on the left-hand side of Fig. 1; the velocity is fixed assuming the density change is negligible; natural convection is ignored as well. This model at constant temperature was first proposed by Wheeler et al. [5], the so-called WBM model, using the minimum of a Gibbs free-energy function. Penrose and Fife [11] and Warren and Boettinger [7] further derived a similar model based on an entropy function, which can be used for nonisothermal growth. However, the temperature effect was ignored in their simulation until

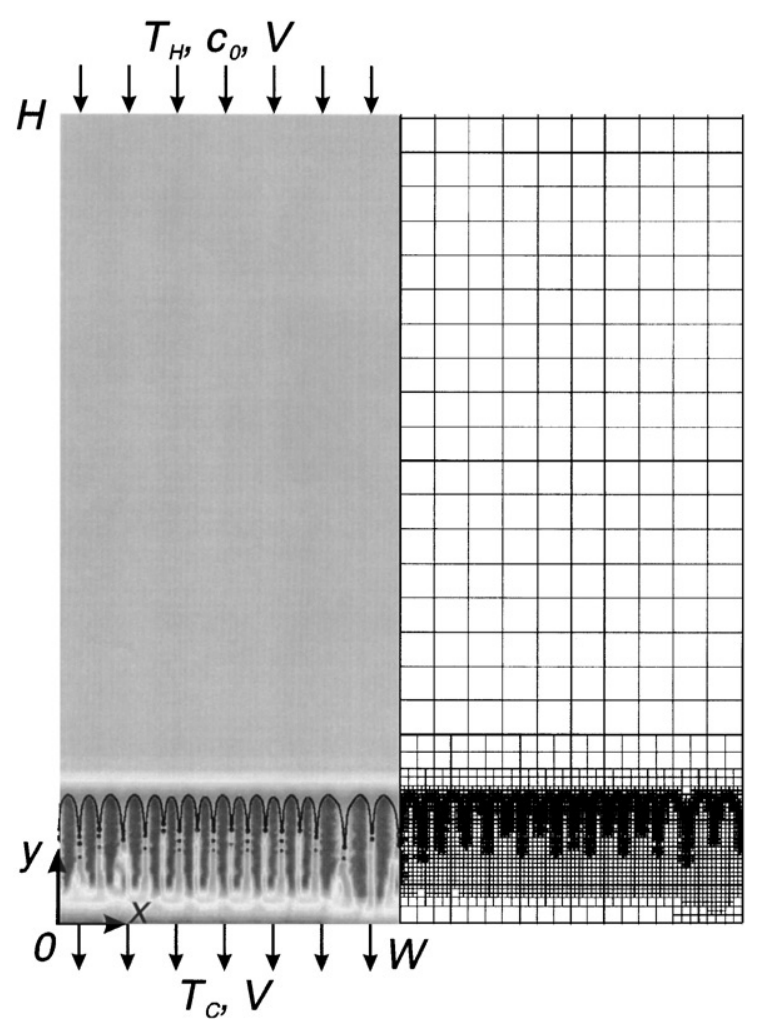

Fig. 1. Cellular directional solidification (left) and adaptive mesh (right) for simulation; the grey area on the left indicates the solute concentration distribution, where the darker area is for the dimensionless concentration of 0.945 and lighter area around it for 1.274. 
recently. Loginova et al. [10] took the energy equation into account, where some ideas due to Caginalp and Xie [12] were introduced. In order to present the governing equations in dimensionless form, the variables are rescaled. The temperature is rescaled to $\theta=\left(T-T_{\text {ref }}\right) / \Delta T$, where $T_{\text {ref }}$ and $\Delta T$ are the reference temperature and temperature difference, respectively. The concentration (atomic fraction) $c$ is rescaled by $c_{0}$ to $c^{*}$, where $c_{0}$ is the far field concentration. The length, in terms of the coordinates $x$ and $y$, is rescaled by $l$, the time $t$ by $l^{2} / D_{\mathrm{L}}$ to $t^{*}$, and the velocity $V$ by $D_{\mathrm{L}} / l$ to $V^{*}$, where $l$ is a characteristic length and $D_{\mathrm{L}}$ the solute diffusivity in the melt. In other words, $l^{2} / D_{\mathrm{L}}$ is the characteristic time and $D_{\mathrm{L}} / l$ is the characteristic velocity. The variables having a superscript asterisk are all dimensionless based on the characteristic variables. The phase filed variable $\phi$ is set to be 1 in liquid and 0 in solid, while 0.5 at the interface. Then, the governing equations can be represented in dimensionless form

$$
\begin{aligned}
\tilde{C}^{*} \frac{\partial \theta}{\partial t^{*}}-V^{*} \frac{\partial \theta}{\partial y^{*}}+\left(\frac{30 g(\phi)}{\tilde{S} t}\right) \frac{\partial \phi}{\partial t^{*}}=\nabla \cdot \tilde{L} e \nabla \theta & \\
\frac{\partial c^{*}}{\partial t^{*}}-V^{*} \frac{\partial c^{*}}{\partial y^{*}}= & \nabla \cdot D^{*}\left[\nabla c+c^{*}\left(1-c_{0} c^{*}\right)\right. \\
& \left.\times\left(S_{\mathrm{B}}^{*}-S_{\mathrm{A}}^{*}\right) \nabla \phi\right], \\
\frac{\partial \phi}{\partial t^{*}}-V^{*} \frac{\partial \phi}{\partial y^{*}}= & \tilde{M}_{\phi}^{*} \tilde{\epsilon}^{* 2}\left[\nabla \cdot\left(\eta^{2} \nabla \phi\right)-\frac{\partial}{\partial x}\left(\eta \eta_{\beta} \frac{\partial \phi}{\partial y}\right)\right. \\
& \left.+\frac{\partial}{\partial y}\left(\eta \eta_{\beta} \frac{\partial \phi}{\partial x}\right)\right]-\tilde{M}_{\phi}^{*} \tilde{S}^{*}
\end{aligned}
$$

where $\tilde{C}^{*}$ is the normalized heat capacity being scaled by the value at $c_{0}$, i.e., $\tilde{C}^{*}=\tilde{C} / \tilde{C}\left(c_{0}\right)$. The variable with a tilde is the concentration-weighted average. For example, the heat capacity $\tilde{C}$ is defined by

$\tilde{C}=(1-c) C_{\mathrm{A}}+c C_{\mathrm{B}}$,

where $C_{\mathrm{A}}$ and $C_{\mathrm{B}}$ are the heat capacity of the solvent (A) and the solute (B). Inside the twophase region of the interface, the properties are obtained by using a weighting function $p(\phi)$, which is obtained from a double well function $g(\phi)$ defined by $g(\phi)=\phi^{2}(1-\phi)^{2}$. The weighting function $p(\phi)$ for the averaged physical properties of the solid/liquid mixture is chosen such that $p^{\prime}(\phi)=30 g(\phi)$. For example, the normalized diffusivity of the solution is given by

$$
D^{*}=D / D\left(c_{0}\right)=\left[D_{\mathrm{S}}+p(\phi)\left(D_{\mathrm{L}}-D_{\mathrm{S}}\right)\right] / D_{\mathrm{L}},
$$

where the diffusivities have been assumed not affected by the solute concentration, i.e., $D=\tilde{D}$. The Stefan number $\tilde{S} t \equiv \tilde{C}\left(c_{0}\right) \Delta T / \Delta \tilde{H}$, where $\Delta \tilde{H}$ is the heat of fusion, while the Lewis number $\tilde{L} e \equiv$ $\tilde{\alpha} / D_{\mathrm{L}}$, where $\tilde{\alpha} \equiv \tilde{K} / \tilde{C}\left(c_{0}\right)$ is the concentrationaveraged thermal diffusivity. In addition, $S_{\mathrm{A}}^{*}$ and $S_{\mathrm{B}}^{*}$ are the normalized entropy of $\mathrm{A}$ and $\mathrm{B}$, respectively, being scaled by $R / V_{\mathrm{m}}$, i.e., $S_{i}^{*}=$ $S_{i} V_{\mathrm{m}} / R(i=\mathrm{A}$ or $\mathrm{B}) ; V_{\mathrm{m}}$ is the molar volume and $R$ the gas constant. The entropies of $\mathrm{A}$ and $\mathrm{B}$ are defined as follows:

$$
\begin{aligned}
& S_{\mathrm{A}}(\phi, T)=W_{\mathrm{A}} g^{\prime}(\phi)+p^{\prime}(\phi) \Delta H_{\mathrm{A}}\left(\frac{1}{T}-\frac{1}{T_{\mathrm{m}}^{\mathrm{A}}}\right), \\
& S_{\mathrm{B}}(\phi, T)=W_{\mathrm{B}} g^{\prime}(\phi)+p^{\prime}(\phi) \Delta H_{\mathrm{B}}\left(\frac{1}{T}-\frac{1}{T_{\mathrm{m}}^{\mathrm{B}}}\right),
\end{aligned}
$$

where $W_{\mathrm{A}}$ and $W_{\mathrm{B}}$ are constants and $T_{\mathrm{m}}^{\mathrm{A}}$ and $T_{\mathrm{m}}^{\mathrm{B}}$ are the melting points of $\mathrm{A}$ and $\mathrm{B}$, respectively; $\Delta H_{\mathrm{A}}$ and $\Delta H_{\mathrm{B}}$ are the heats of fusion per volume. Again, in Eq. (3) $\tilde{S}^{*}$ is the concentration-averaged entropy (normalized).

The anisotropic function $\eta$ in Eq. (3) is defined for the four-fold symmetry as

$\eta=1+\gamma \cos 4 \beta$,

where $\gamma$ is the intensity of the anisotropy and $\beta=$ $\tan ^{-1}[(\partial \phi / \partial y) /(\partial \phi / \partial x)]$ determining the growth orientation of the cell. In this study, we have purposely chosen $(100)$ is in the $x$-direction and $\left(\begin{array}{lll}0 & 1 & 0\end{array}\right)$ in the $y$-direction. Finally, the dimensionless mobility function $\tilde{M}_{\phi}^{*}$ being scaled by $D_{\mathrm{L}} V_{\mathrm{m}} /\left(R l^{2}\right)$ is taken from the average of $M_{i}=$ $T_{\mathrm{m}}^{i^{2}} \beta_{i} /\left(6 \sqrt{2} \Delta H_{i} \delta_{i}\right), i=\mathrm{A}$ or $\mathrm{B}$, where $\beta_{i}$ is the kinetic coefficient and $\delta_{i}$ the interface thickness, which are assumed to be the same for A and B here. Similarly, $\tilde{\varepsilon}^{* 2}$ is a dimensionless parameter being rescaled by $l^{2}$. For each component, $\varepsilon_{i}^{2}=$ $6 \sqrt{2} \sigma_{i} \delta_{i} / T_{\mathrm{m}}^{i}$ where $\sigma_{i}$ is the interfacial energy. All the parameters chosen are the same as those in Ref. [6], which are similar to the ones used in WBM model.

To solve these equations, an adaptive finite volume method has been used. The detailed 
implementation of the scheme can be found elsewhere [13,14]. The simulation starts from a planar interface in a large slender domain, as shown in Fig. 1; the height $H$ in the $y$-direction is larger in order to cover the thermal and solutal boundary layers. On the other hand, the width $W$ in the $x$-direction is chosen such that enough cells can be considered. On the both sides of the domain, the symmetry (no flux) condition is used. On the top boundary, the concentration $\left(c^{*}=1\right)$ and temperature $(\theta=1)$ are given, while at the lower boundary condition, only the temperature $(\theta=0)$ is given. However, the concentration at the lower boundary is set by the exit condition with its own concentration, i.e., $\partial c^{*} / \partial y^{*}=0$ When a steady state is reached, the average concentration (normalized) at the exit boundary is equal to 1 . The initial conditions for the phase field variable is given by

$\phi=\frac{1}{2}\left[1+\tanh \left(\frac{y^{*}-y_{0}^{*}}{2 \sqrt{2}}\right)\right]$,

where $y_{0}^{*}$ is the initial interface position. The concentration is set by a step function as

$c^{*}= \begin{cases}1, & \phi \geqslant 0.5, \\ 0.86, & \phi<0.5 .\end{cases}$

The initial position can be given exactly according to the concentration (0.86) and the linear temperature profile. In addition, $\left(T_{H}-\right.$ $\left.T_{c}\right) / H=G$ and $T=T_{\mathrm{m}, c}$ at the interface, where $G$ is the given thermal gradient and $T_{\mathrm{m}, c}$ the liquidus temperature from the phase diagram. However, for comparison purposes, we have adopted the same initial condition used in Ref. [9] as much possible; the step function in solute concentration at equilibrium is used. In addition, the noise can be introduced if necessary. However, in the present calculation, the morphology will develop even without noises. Nevertheless, by introducing the noise through

$\frac{\partial \phi}{\partial t^{*}} \rightarrow \frac{\partial \phi}{\partial t^{*}}-\tilde{M}_{\phi} \operatorname{ar}(16 g(\phi)) \tilde{S}^{*}$

the steady state can be reached more quickly, where $a$ is the noise intensity and $r$ the random number ranging from -1 to 1 .
A sample mesh during growth is shown on the right-hand side of Fig. 1. One can see that the mesh adapts to the interface shape nicely. As shown, mesh refinement is performed by subdividing uniformly each parent cell into four kid cells. As such, the detailed substructures can also be described nicely if the mesh level is large enough; in Fig. 1, eight levels are used for the mesh; the finest cell size remains to be smaller than $0.6 l$, which needs to be smaller than the interface thickness and the solute boundary layer thickness. It should be pointed out that the solutal boundary layer thickness, estimated by $D / V$, is about $5 \times 10^{-8} \mathrm{~m}$ for the largest speed used here $(2 \mathrm{~cm} / \mathrm{s})$, which is about the value of the interface thickness adopted here. In other words, to have an accurate calculation, the cell size needs to be much smaller than this value. However, in the previous calculations [9], the cell size is only about $4.4 \times 10^{-8} \mathrm{~m}$, which is believed to be inadequate for high-speed calculations. A typical calculation for high speed takes less than one hour of CPU time. However, for low speed, it may take much longer, up to several days, on a PC having a $2 \mathrm{GHz}$ P4-CPU. Two criteria are used here for the refinement. One is based on the phase field variable (refinement at $0.05<\phi<0.95$ ) and one is on the concentration gradient (refinement for $|\nabla c|>0.01)$. In addition, the level difference for adjacent cells is also restricted to one, and this improves significantly the accuracy of the method. By mapping the grid into a quad-tree data structure, the dynamic pointer functions in FORTRAN90 can be fully adopted and this makes the programming straightforward. The implicit Euler scheme is used for time integration and this allows larger integration timesteps to be used. Nevertheless, the limitation for the timesteps is that the interface advancement needs to be inside the refined zone. Otherwise, numerical instability can cause problems. In the present simulation, the CPU time for a growth scales about $W \delta_{c}$, where $\delta_{c}$ is the solutal boundary layer thickness. In other words, the computational cost is about linearly proportional to the area having larger solutal gradients. This is comparable to the performance of the scheme proposed by Provatas et al. [15]. 


\section{Results and discussion}

For comparison purposes, the $\mathrm{Ni} / \mathrm{Cu}$ system used by Warren and Boettinger [9] is considered here. The physical properties and system related parameters are as follows, unless otherwise stated: $W=400 l, H=500 l, y_{0}=368 l, G=2.15 \times 10^{4}$ $\mathrm{K} / \mathrm{cm}, \Delta T=49.5145 \mathrm{~K}, T_{\text {ref }}=1558.06275 \mathrm{~K}, c_{0}=$ 0.4083 (atomic fraction), and 5 levels are used for meshes. All physical properties and phase field parameters related to the alloy properties are given in Ref. [9], where the same kinetic coefficient $\beta=0.35 \mathrm{~cm} / \mathrm{K}$ has been chosen, which is about 570 times smaller than the collision limited growth of metal. Increasing this coefficient requires the decrease of timestep for integration. However, we have examined several values, and found that the steady-state morphology is not affected much, even though the transient behavior is affected much more. The interface thickness $\left(\delta=4.5 \times 10^{-8} \mathrm{~m}\right)$ is also chosen to be about the same as that in Refs. $[9,10]$. Again, for steady state, we do not find any significant difference by reducing this value, except solute trapping. With a larger interface thickness, the maximum value buildup at the interface region is smaller, but the global solutal field remains similar. However, reducing the interface thickness also requires a smaller timestep and this increases the computation time proportionally, especially for the low growth speed. In fact, from Eq. (3) $\tilde{M}_{\phi}^{*} \tilde{\epsilon}^{* 2}$ being proportional to $\beta$ can be regarded is a dimensionless diffusivity for the phase field variable. Decreasing $\beta$, while increasing $\delta$, makes the simulation easier. However, a too large $\delta$ value will lead to large error in the estimation of solute trapping. Therefore, as a trade-off and for the comparison purposes with previous work [9-10], we have chosen $\beta$ and $\delta$ values similar to the ones used by Boettinger and Warren [9] and Loginova et al. [10].

\subsection{Frozen temperature approximation (FTA)}

From the Mullins-Sekerka's analysis, the interface remains flat at low speed (lower than the critical velocity) and high speed (higher than the absolute stability limit). Therefore, with a given thermal gradient $2.15 \times 10^{4} \mathrm{~K} / \mathrm{cm}$ [9], we first carry out calculation for $V=4 \mathrm{~cm} / \mathrm{s}$ without introducing any disturbance $(a=0)$. As shown in Fig. 2a, the interfaces remain flat all time and show very little segregation; the concentration is uniform. Even with a disturbance $(a=0.4)$, the interface gets some ripples and some segregation occurs at the beginning. However, the interface eventually becomes flat and the solution is the same as the one without the disturbance at steady state. If we lower the growth rate slightly to $3 \mathrm{~cm} / \mathrm{s}$, some patterns form. Fig. 3 shows the comparison of the calculated time evolution of the interface and

(a)
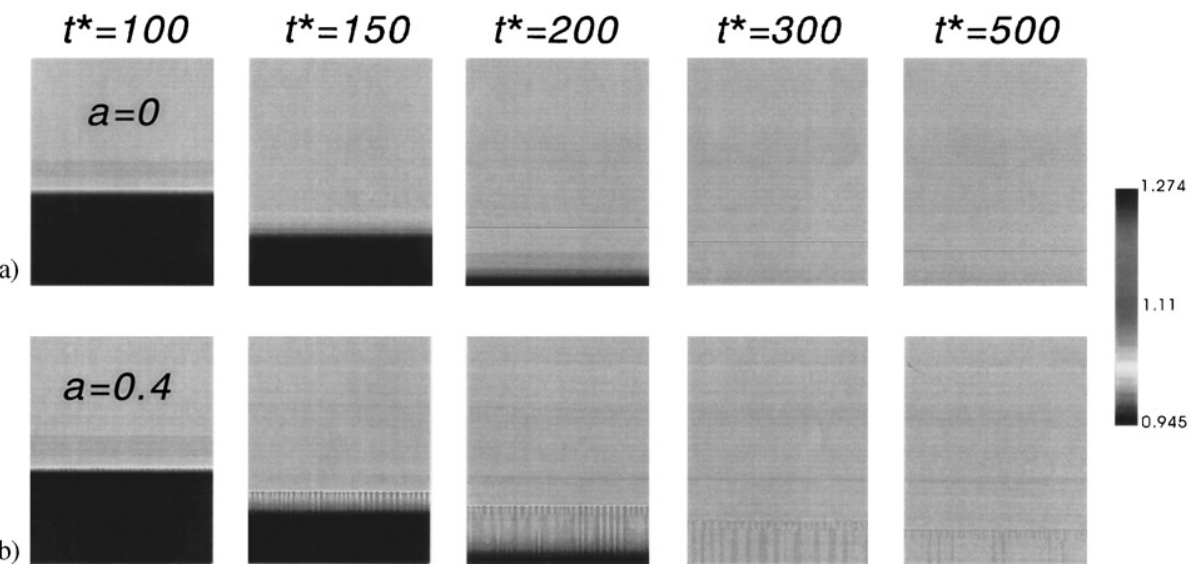

Fig. 2. Time evolution of interface and solutal fields for $V=4 \mathrm{~cm} / \mathrm{s}$ : (a) without artificial noises $(a=0)$; (b) with artificial noises $(a=4)$; the grey shading indicates the solute concentration distribution. 


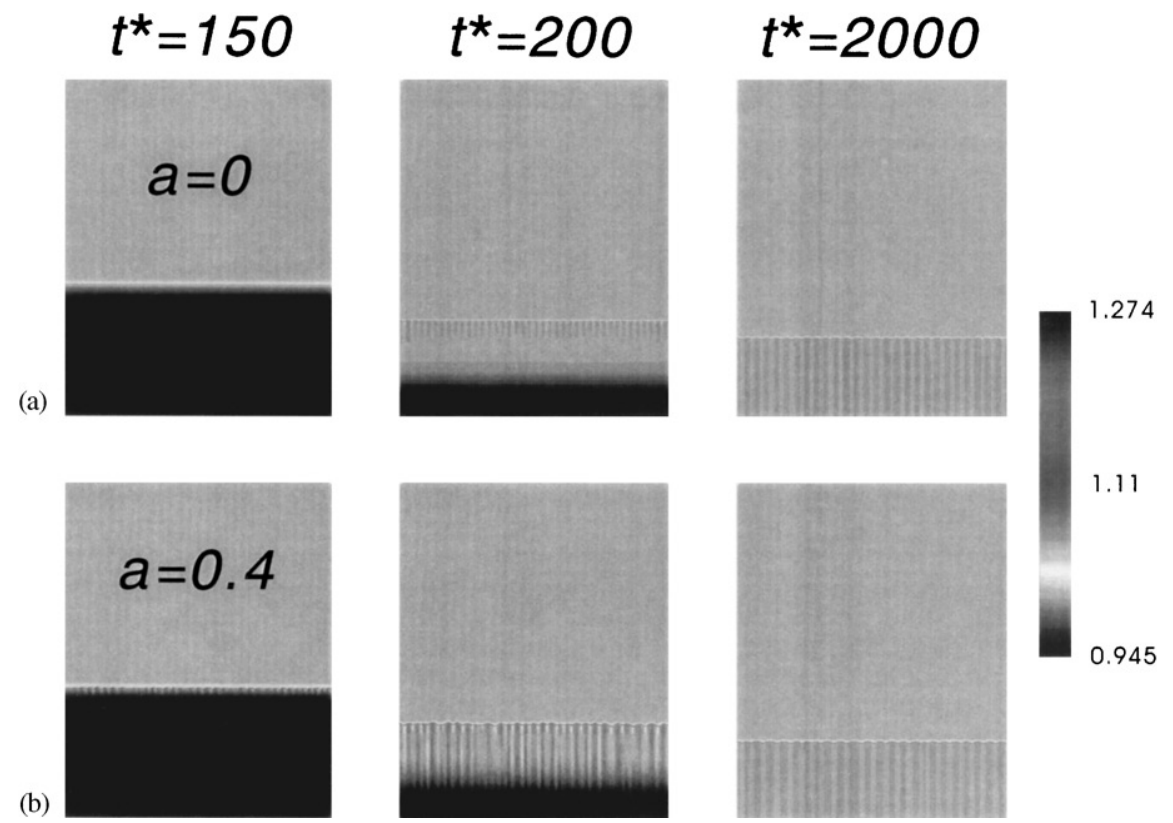

Fig. 3. Time evolution of interface and solutal fields for $V=3 \mathrm{~cm} / \mathrm{s}$ : (a) without artificial noises $(a=0)$; (b) with artificial noises $(a=4)$; the grey shading indicates the solute concentration distribution.

concentration without Fig. 3(a) and with Fig. 3(b) artificial noises. As shown, beside some difference at the transient state, the steady pattern and concentration fields remain about the same. Therefore, unlike that in free dendritic growth, the noises affect very little the final steady state of the growth here. Therefore, the absolute stability limit is between 3 and $4 \mathrm{~cm} / \mathrm{s}$. As will be discussed shortly, this value is way below the MullinsSekerka prediction.

The steady-state patterns and solute fields for different speeds are summarized in Fig. 4. Apparently, the cell wavelength decreases with the increasing growth rate. For the cases with a lower growth rate, such as 0.0625 or $0.125 \mathrm{~cm} / \mathrm{s}$, the domain width ( $W=400 l$ ) seems to be too small to cover enough cells for simulation. In other words, the symmetry assumption at the side boundaries starts to break down. In addition, in our calculations, we have not yet found any chaotic pattern formation, which was observed by Boettinger and Warren, at the speed of $0.0625 \mathrm{~cm} / \mathrm{s}$.

In addition, with the lower growth rate, the solute boundary layer thickness also increases, which can be estimated by $D_{\mathrm{L}} / V$. In other words, for a speed of $0.01 \mathrm{~cm} / \mathrm{s}$, the boundary layer thickness is up to $1500 l$. This indicates that for the lower speed, the simulation requires not only larger width, but also a much longer length. Therefore, for $V=0.03125 \mathrm{~cm} / \mathrm{s}$, we have to increase the width to $2000 \mathrm{l}$ and length to $5000 \mathrm{l}$ for calculation; 9 levels of grid are used. The time evolution of the interface and concentration (at later stage) is shown in Fig. 5. Again, the time for reaching a steady state is also much longer being larger than $t^{*}=70,000$, which also makes the calculation difficult. For the same domain size, it requires about $2000 \times 5000$, i.e., $10^{7}$, cells for a structured mesh. However, by using the adaptive grid, $10^{5}$ cells are adequate, which is about two orders of magnitude smaller. For $V=0.015625 \mathrm{~cm} / \mathrm{s}$, the result is similar, but the dimensionless time to steady state is longer than 200,000.

For a speed of $0.0078125 \mathrm{~cm} / \mathrm{s}$ or lower, we have not yet found any pattern development. The interface remains flat at all time. This indicates that we have crossed the lower critical boundary 

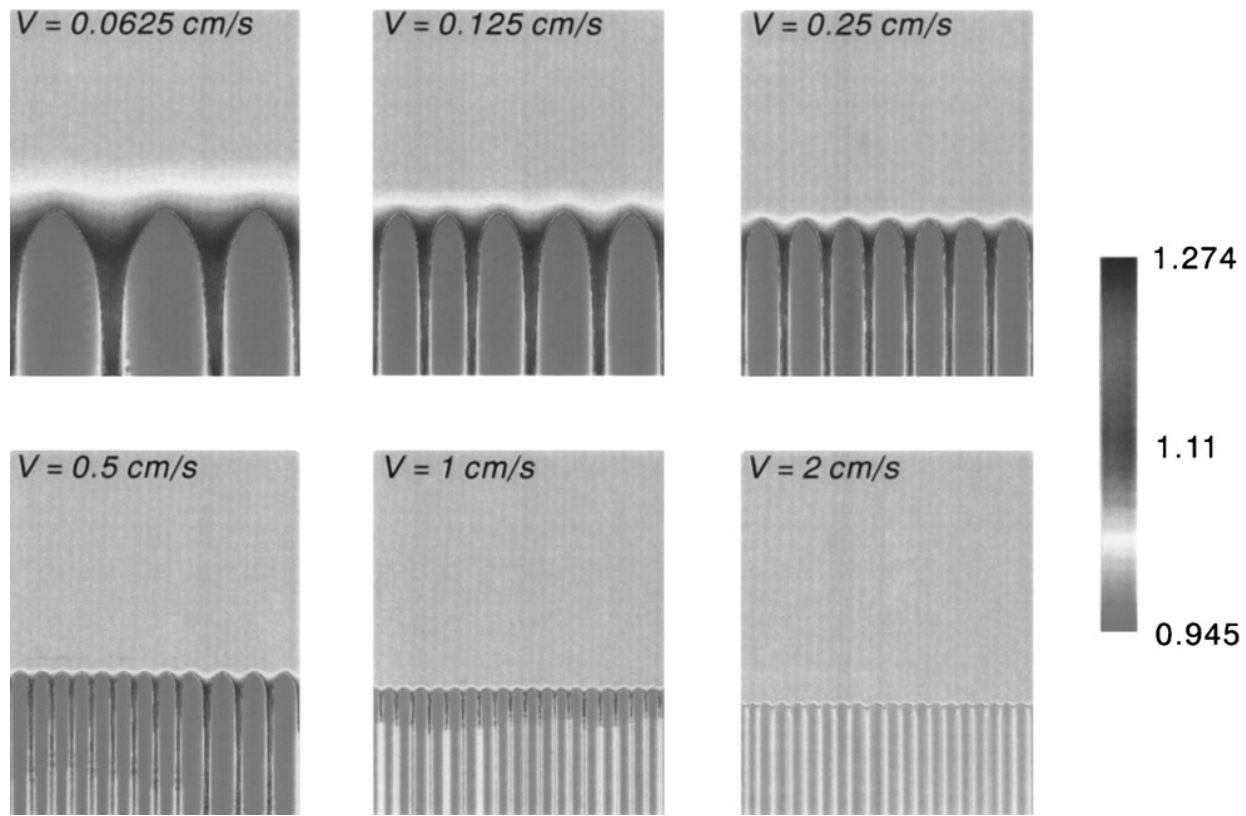

Fig. 4. Steady-state cellular structures and solutal fields at different growth speeds; the grey shading indicates the solute concentration distribution.

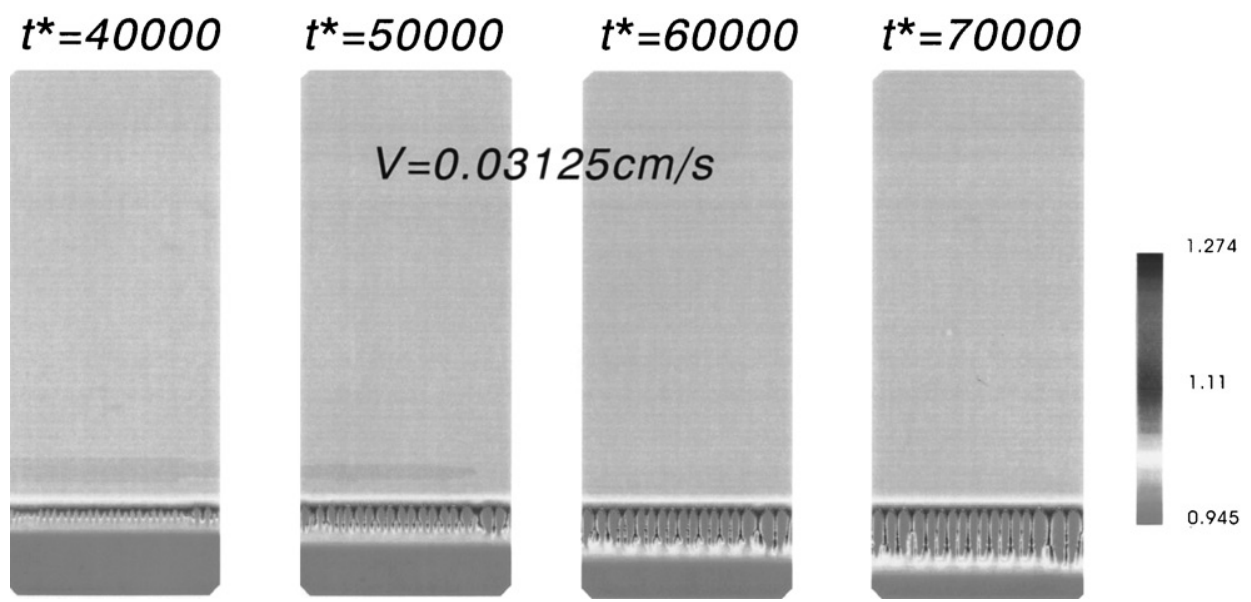

Fig. 5. Time evolution of interface and solutal fields for $V=0.03125 \mathrm{~cm} / \mathrm{s} ; a=0.4$. The grey shading indicates the solute concentration distribution.

for the planar interface. The summary of the calculations is put into the stability diagram obtained from the Mullins-Sekerka theory (MSloop), as shown in Fig. 6, where the data by Warren and Boettinger [9] are put together for comparison. As shown, the agreement is reason- ably good. In addition, the trend pretty much satisfies the scaling law of $V \lambda^{2}=$ constant [16], where $\lambda$ is the tip radius or cell wavelength. In Fig. 6, wavelength is obtained by taking an average of the cellular spacing after a steady state is reached. Both our and previous calculations 


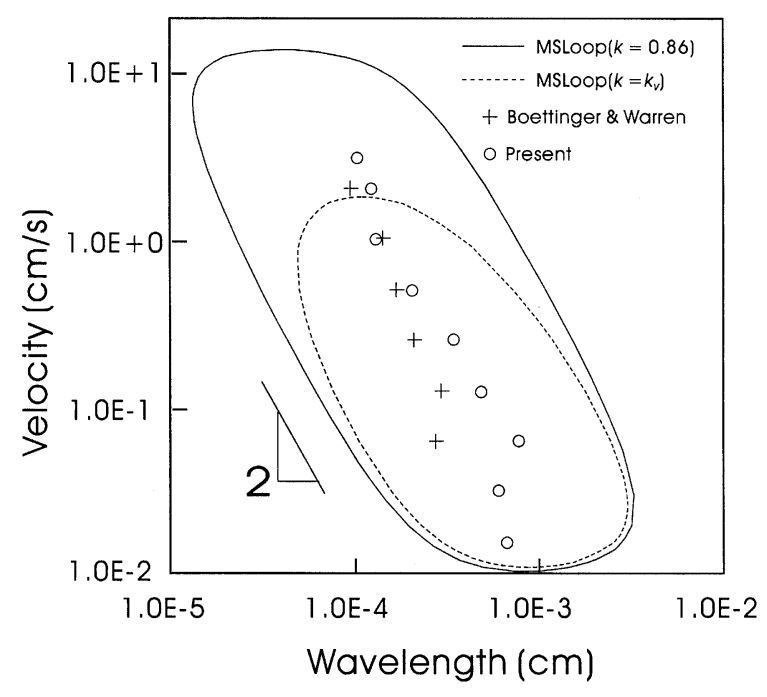

Fig. 6. Summary of the calculated points on the MullinsSekerka instability loop (MS-loop); the dashed line is calculated using the velocity-dependent segregation coefficient $k_{v}$.

show a much lower absolute stability limit. This could be the cause of solute trapping, which will be discussed in the next section. We also put the MSloop using the velocity-dependent segregation coefficient in Fig. 6 (dashed line) for comparison. As shown, the agreement of the absolute stability limit is greatly improved.

\subsection{Segregation coefficients and solute trapping}

From the previous calculations, if we take the concentration along the cell tip, the segregation behavior can be better understood, as illustrated in Fig. 7a. We do not put the result of lower speeds because the solutal boundary layer is much thicker. As shown from Fig. 7a, the peak concentration increases with decrease in growth rate. The ratio of solute concentration in the solid $\left(C_{\mathrm{S}}\right)$ and liquid $\left(C_{\mathrm{L}}\right)$ also increases with the decreasing speed. Furthermore, we can thus define a velocity-dependent segregation coefficient $k_{v}=C_{\mathrm{S}} / C_{\mathrm{L}}$. At equilibrium and zero interface thickness, $k_{v}$ is equal to $k$ and is independent of the growth rate, where $k$ is the equilibrium segregation coefficient ( $k=0.86$ here). The increase of $k_{v}$ is due to the solute trapping in the diffusive interface. Aziz [17] and Aziz and Kaplan [18] have derived the segregation coefficient $k_{v}$, which is as a function of interface diffusion velocity $V_{\mathrm{D}}$ is given by

$k_{v}=\frac{k+V / V_{\mathrm{D}}}{1+V / V_{\mathrm{D}}}$.

If we assume that $V_{\mathrm{D}}$ is unknown and can be obtained by the best fitting of above equation to the simulated data, as shown in Fig. 7b, we get $V_{\mathrm{D}}=0.767 \mathrm{~cm} / \mathrm{s}$. Ahmad et al. [19] also derived $V_{\mathrm{D}}$ based on a simple model, and they came out with a formula assuming $D_{\mathrm{S}} \ll D_{\mathrm{L}}$

$V_{\mathrm{D}}=0.207 \frac{D_{\mathrm{L}} \ln (l / k)}{\delta(1-k)}$,

where $\delta$ is interface thickness $\left(4.5 \times 10^{-8} \mathrm{~m}\right)$. Based on this, $V_{\mathrm{D}}=0.484 \mathrm{~cm} / \mathrm{s}$, which is lower than ours. However, Boettinger and Warren [9] obtained an even larger value at $V_{\mathrm{D}}=1.03 \mathrm{~cm} / \mathrm{s}$. Therefore, our calculated value is still in a reasonable range as compared with previous works.

In addition, from Eq. (13) it is also clear that the interface diffusion velocity increases with the decreasing interface thickness. In other words, when the interface thickness is small, the diffusion velocity increases and $k_{v}$ is closer to $k$. This indicates that the solute trapping is reduced with a thinner interface thickness. Although the interface thickness used here is still much larger than the real one, the solute trapping behavior is pretty much predicted. Furthermore, the predicted absolute limit in Fig. 6 is also reasonable. We have summarized our results in Table 1, where the results by Boettinger and Warren [9] are included for comparison.

\subsection{Non-isothermal effects}

In the previous calculations, FTA is used, so that the energy equation is not used for simulation. For truly non-isothermal cases, we have to include the energy equation, Eq. (1), in our simulation. In other words, Eqs. (1)-(3) need to be solved simultaneously. Once the energy is included, the heat of fusion released during solidification can be considered. As a result, the thermal gradient in the melt side will decrease, while in the solid side increase. Because the thermal gradient in the simulation is very large being $2.15 \times 10^{4} \mathrm{~K} / \mathrm{cm}$, it 

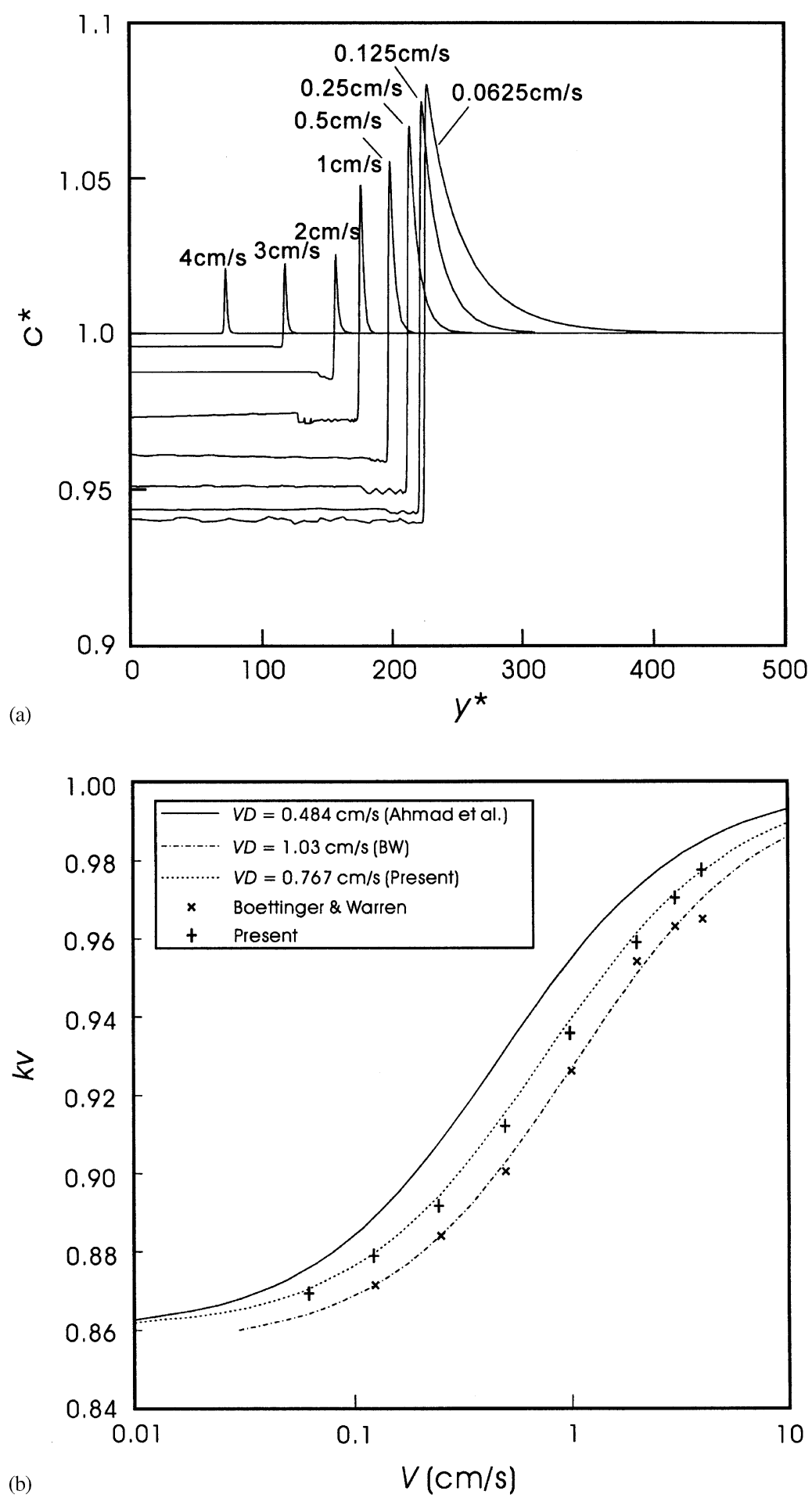

Fig. 7. (a) Solute distribution along the dendrite tip at different growth speeds; and (b) calculated segregation coefficients as a function of growth rate. 
Table 1

Comparison of calculated values with Boettinger and Warren [9]

\begin{tabular}{llllllll}
\hline & $V(\mathrm{~cm} / \mathrm{s})$ & $\lambda(\mathrm{cm})$ & $T_{\text {tip }}(\mathrm{K})$ & $C_{\mathrm{L}, \text { tip }}$ & $C_{\mathrm{S} \text {,tip }}$ & $k$ & $V \lambda^{2}(\mathrm{~cm} / \mathrm{s})$ \\
\hline Boettinger and Warren [9] & 0.0625 & $2.56 \times 10^{-4}$ & $1576.2 \pm 3$ & - & - & - & $4.1 \times 10^{-9}$ \\
& 0.125 & $2.84 \times 10^{-4}$ & 1578.7 & 0.442 & 0.385 & 0.872 & $1.01 \times 10^{-8}$ \\
& 0.25 & $2.04 \times 10^{-4}$ & 1578.1 & 0.438 & 0.387 & 0.884 & $1.04 \times 10^{-8}$ \\
& 0.5 & $1.54 \times 10^{-4}$ & 1576.8 & 0.434 & 0.391 & 0.901 & $1.19 \times 10^{-8}$ \\
& 1 & $1.32 \times 10^{-4}$ & 1574.9 & 0.425 & 0.394 & 0.927 & $1.74 \times 10^{-8}$ \\
& 2 & $8.71 \times 10^{-5}$ & 1569.8 & 0.422 & 0.403 & 0.955 & $1.52 \times 10^{-8}$ \\
Present & 3 & Planar & 1563.5 & 0.424 & 0.408 & 0.964 & - \\
& 4 & Planer & 1556.1 & 0.423 & 0.408 & 0.966 & - \\
& & & & & & \\
& 0.0625 & $7.68 \times 10^{-4}$ & 1580.0 & 0.442 & 0.384 & 0.869 & $3.68 \times 10^{-8}$ \\
& 0.125 & $4.61 \times 10^{-4}$ & 1579.9 & 0.438 & 0.385 & 0.879 & $2.65 \times 10^{-8}$ \\
& 0.25 & $3.29 \times 10^{-4}$ & 1579.1 & 0.435 & 0.388 & 0.892 & $2.71 \times 10^{-8}$ \\
& 0.5 & $1.92 \times 10^{-4}$ & 1577.6 & 0.431 & 0.393 & 0.912 & $1.84 \times 10^{-8}$ \\
& 1 & $1.21 \times 10^{-4}$ & 1575.5 & 0.426 & 0.398 & 0.936 & $1.47 \times 10^{-8}$ \\
& 2 & $1.15 \times 10^{-4}$ & 1573.5 & 0.419 & 0.402 & 0.959 & $2.65 \times 10^{-8}$ \\
& 3 & $9.8 \times 10^{-5}$ & 1569.7 & 0.417 & 0.405 & 0.971 & $2.88 \times 10^{-8}$ \\
& 4 & Planer & 1565.2 & 0.417 & 0.408 & 0.978 & - \\
\hline
\end{tabular}

is not realistic to consider a large domain having a reasonable temperature at the top and the bottom. Instead, we have chosen the same domain size as the previous examples. It should be pointed out that including the energy equation does not increase the computational effort too much. However, since the thermal diffusivity $\left(2.7 \times 10^{-5} \mathrm{~m}^{2} / \mathrm{s}\right)$ is several orders larger than solutal diffusivity $\left(10^{-9} \mathrm{~m}^{2} / \mathrm{s}\right)$, the far field boundary can be affected quickly. For a realistic simulation, the domain needs to be large enough. Due to the restriction of the large thermal gradient, and for the comparison with the theory, the upper and lower temperatures are kept the same. For a planar interface, at steady state, the temperature and interface can be determined analytically

$\theta=1+\left(\theta_{\mathrm{m}, \mathrm{c}}-1\right) \frac{1-\mathrm{e}^{-P e\left(H-y^{*}\right)}}{1-\mathrm{e}^{-P e\left(H-y_{i}^{*}\right)}} \quad$ when $y^{*}>y_{i}^{*}$,

$\theta=\theta_{\mathrm{m}, \mathrm{c}} \frac{1-\mathrm{e}^{-P e\left(H-y^{*}\right)}}{1-\mathrm{e}^{-P e\left(H-y_{i}^{*}\right)}} \quad$ when $y^{*}<y_{i}^{*}$,

where the interface position $y_{i}^{*}$ can be determined from the Stefan condition

$\frac{\left(\theta_{\mathrm{m}, \mathrm{c}}-1\right)}{1-\mathrm{e}^{-P e\left(y_{i}^{*}-H\right)}}=\frac{\theta_{\mathrm{m}, \mathrm{c}}}{1-\mathrm{e}^{- \text {Pe } y_{i}^{*}}}-S t^{-1}$, where $P e \equiv V l / \tilde{\alpha}$ is the Peclect number. For a small $P e$ number, which is usually true for the metallic system at a normal growth rate, the temperature profiles in the melt and the solid are essentially linear. The interface energy balance at the interface also implies $G_{\mathrm{S}}=G_{\mathrm{L}}+P e S t^{-1}$, where $G_{\mathrm{S}}$ and $G_{\mathrm{L}}$ are the thermal gradient at the solid and liquid, respectively. Our calculated thermal profiles are in good agreement with previous analytical results at all speeds. The calculated thermal profiles $\left(\theta\right.$ vs. $\left.y^{*}\right)$ at $2 \mathrm{~cm} / \mathrm{s}$ at two different thermal gradients are shown in Fig. 8, where the initial temperature distribution (a straight line) is included for comparison. As shown, to get a larger effect of latent heat, the thermal gradient needs to be lower. Unfortunately, at the lower thermal gradient at the same speed, the cell wavelength is larger; the MS-loops at different thermal gradients are included in the same figure for reference. The increase of the cell wavelength is linearly proportional the computational cost, and this indicates that almost one order of magnitude higher in CPU time for $2.15 \times 10^{3} \mathrm{~K} / \mathrm{cm}$ and two orders for $2.15 \times 10^{2} \mathrm{~K} /$ $\mathrm{s}$ (for $2 \mathrm{~cm} / \mathrm{s}$ ). In other words, the domain width needs to be increased about 50 times for $2.15 \times 10^{2} \mathrm{~K} / \mathrm{cm}$ because of the much longer cell wavelength. 


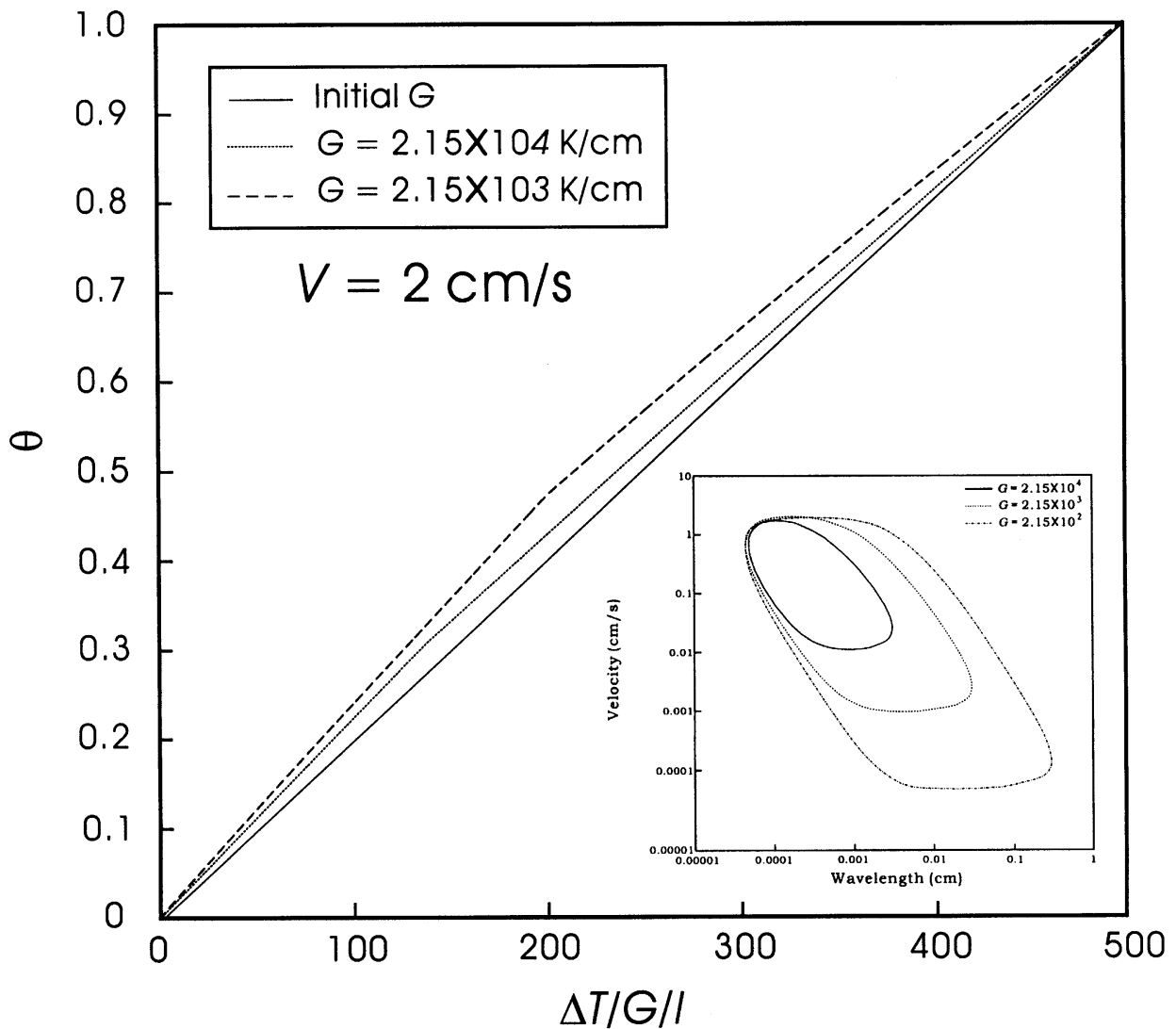

Fig. 8. Calculated thermal distributions at two different initial thermal gradients at $V=2 \mathrm{~cm} / \mathrm{s}\left(\Delta T / G / l\right.$ is equivalent to $\left.y^{*}\right)$; the lower right corner shows the MS loops at different thermal gradients.

Table 2

Comparison with FTA/non-FTA calculated results

\begin{tabular}{lllll}
\hline$V(\mathrm{~cm} / \mathrm{s})$ & $\lambda(\mathrm{cm})$ & $d_{\mathrm{g}}^{*}(\mathrm{~cm})$ & $T_{\text {tip }}(\mathrm{K})$ & $y_{\text {i,tip }}(l)$ \\
\hline 0.5 & $1.92 \times 10^{-4} / 1.92 \times 10^{-4}$ & $6.24 \times 10^{-4} / 5.36 \times 10^{-4}$ & $1577.6 / 1577.6$ & $194.253 / 193.084$ \\
1 & $1.21 \times 10^{-4} / 1.29 \times 10^{-4}$ & $1.74 \times 10^{-4} / 1.88 \times 10^{-4}$ & $1575.5 / 1575.5$ & $175.773 / 166.334$ \\
2 & $1.15 \times 10^{-4} / 1.21 \times 10^{-4}$ & $3.28 \times 10^{-5} / 4.06 \times 10^{-5}$ & $1573.5 / 1573.5$ & $155.136 / 140.181$ \\
3 & $9.8 \times 10^{-5} / 8.5 \times 10^{-5}$ & $5.98 \times 10^{-6} / 5.26 \times 10^{-6}$ & $1569.7 / 1569.7$ & $117.196 / 98.474$ \\
4 & Planer & 0 & $1565.2 / 1565.2$ & $72.298 / 55.997$ \\
\hline
\end{tabular}

$d_{\mathrm{g}}^{*}$ : the glove depth.

The time evolution of the interface and solute fields for both FTA and non-FTA results are illustrated in Fig. 9. As shown, except the lower interface positions due to the release of latent heat, the results are very close. Further comparison is shown in Table 2. The difference, except in the interface position, is within simulation errors. This seems to further validate the reasonable assumption of FTA. However, this could be misleading. In this simulation, also that in Ref. [9], the thermal gradient is very high being $2.15 \times 10^{4} \mathrm{~K} / \mathrm{cm}$, so that the effect of latent heat is small. With smaller thermal gradients, say $2.15 \times 10^{2} \mathrm{~K} / \mathrm{cm}$, the effect could be much more. However, it requires much 

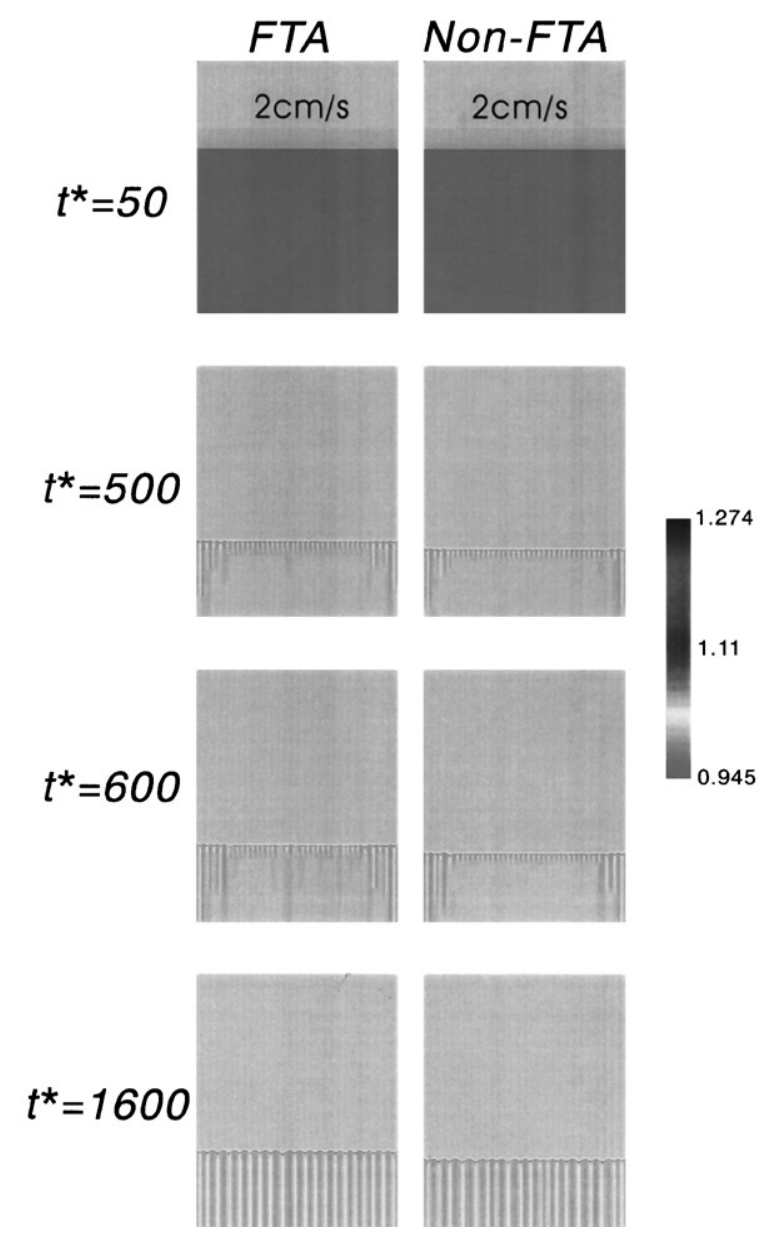

Fig. 9. Comparison of time evolution of interface and solutal fields of FTA and non-FTA simulations; the grey shading indicates the solute concentration distribution.

more CPU time to get a result, because as just mentioned, the domain size needs to be increased significantly. Therefore, for a realistic simulation, one would need a larger interface thickness, and this implies that a new phase field model, e.g. with anti-solute trapping, that is less sensitive to the interface thickness is desirable.

\section{Conclusions and comments}

We have presented an efficient adaptive phase simulation for directional solidification. The morphological development and solute trapping from planar to cellular growth, as well as the absolute stability limit, are illustrated with and without the FTA. With the adaptive mesh, the large solutal boundary layer and wavelength at low speed can be simulated, while keeping small enough cells for the diffusive interface. Such a simulation could be much more difficult to carry out by using a structured mesh. Our results agree reasonably well with the previous studies. However, due to the large thermal gradient used here, the effect of latent heat is not significant, even at high growth rate. However, the computation at lower thermal gradients, which result in much larger cell wavelength and solutal boundary layer, remains a great challenge for simulation, even with the current adaptive scheme. Not only is the large domain required, but also the time constant is inevitably large for a realistic simulation. Nevertheless, the present simulation approach has shed some light for a realistic simulation, and with a more powerful computer, such a realistic simulation could be feasible.

\section{Acknowledgements}

CWL would like to thank Prof. N. Imaishi for his generous hospitality for the author's threemonth stay in Kyushu University. The visiting Professorship to CWL sponsored by Kyushu University is highly appreciated. This research is sponsored by the National Science Council of the Republic of China.

\section{References}

[1] W.W. Mullins, R.F. Sekerka, J. Appl. Phys. 35 (1964) 444.

[2] J.A. Warren, J.S. Langer, Phys. Rev. E 47 (1993) 2702.

[3] K. Tsiveriotis, R.A. Brown, Int. J. Numer. Methods Fluids 16 (1993) 827.

[4] H.S. Udaykumar, H.C. Kan, W. Shyy, T.S.T. Roger, J. Comput. Phys. 137 (1997) 366.

[5] A.A. Wheeler, W.J. Boettinger, G.B. McFadden, Phys. Rev. A 45 (1992) 7424.

[6] A.A. Wheeler, W.J. Boettinger, G.B. McFadden, Phys. Rev. E 47 (1993) 1893.

[7] J.A. Warren, W.J. Boettinger, Acta Metall. Mater. 43 (1995) 689. 
[8] W.J. Boettinger, J.A. Waren, Metall. Mater. Trans. 27A (1996) 657.

[9] W.J. Boettinger, J.A. Warren, J. Crystal Growth 200 (1999) 583.

[10] I. Loginova, G. Amberg, J. Argen, Acta Mater. 49 (2001) 573.

[11] O. Penrose, P.C. Fife, Physica D 43 (1990) 44.

[12] G. Caginalp, W. Xie, Phys. Rev. A 48 (1993) 1897.

[13] C.W. Lan, C.C. Liu, C.M. Hsu, J. Comput. Phys. 178 (2002) 464
[14] C.W. Lan, C.M. Hsu, C.C. Liu, Y.C. Chang, Phys. Rev. E 65 (2002) \#061601.

[15] N. Provatas, N. Goldendeld, J. Dantzig, Phys. Rev. Lett. 83 (22) (1999) 4496.

[16] J.S. Langer, H. Muller-Krumbhaar, Acta. Metall. 42 (1977) 11.

[17] M.J. Aziz, J. Appl. Phys. 53 (1982) 1158.

[18] M.J. Aziz, T. Kaplan, Acta. Metall. 36 (1988) 2335.

[19] N.A. Almad, A.A. Wheeler, W.J. Boettinger, G.B. McFadden, Phys. Rev. E 58 (1988) 3436. 